\title{
Endothelial integrins and angiogenesis: not so simple anymore
}

\author{
Dean Sheppard \\ Department of Medicine, Sandler Center for Basic Research in Asthma and the Lung Biology Center, University of California, San Francisco, \\ San Francisco, California, USA
}

J. Clin. Invest. 110:913-914 (2002). doi:10.1172/JCI200216713.

The rapid growth and precise spatial organization of new blood vessels are central features of organ development, tissue repair, and neoplasia. Like many other processes that require tight spatial regulation, blood vessel formation is critically dependent on extracellular signals that are spatially restricted by their association with components of the ECM. Once regarded as simply a structural scaffold for the maintenance of tissue integrity, the ECM has emerged over the past decade as a highly dynamic repository of positional signals. These signals include matrix-associated growth factors, cytokines, and proteases as well as the ECM proteins, such as collagens, laminins, and fibronectin, which contribute to structural integrity.

Integrins are heterodimeric transmembrane proteins that function principally as receptors for spatially restricted extracellular ligands, including most of the structural components of the ECM and several matrix-associated growth factors and proteases (1). It is therefore not surprising that several integrins have been implicated in vascular development and angiogenesis (2-5). However, there is controversy about the principal integrins involved in this regulation in vivo as well as the mechanisms by which these integrins regu-

\footnotetext{
Address correspondence to: Dean Sheppard, Lung Biology Center, Box 0854, University of California, San Francisco, San Francisco, California 94110, USA.

Phone: (415) 206-5901; Fax: (415) 206-4123;

E-mail: deans@itsa.ucsf.edu.

Conflict of interest: No conflict of interest has been declared.

Nonstandard abbreviations used: protein kinase A (PKA).
}

late normal and pathological blood vessel development.

New data from Kim et al. (6) in this issue of the JCI suggest that a resolution of this controversy may now be emerging. The authors convincingly show that in endothelial cells activated by the growth factor bFGF, inhibitors of the integrins $\alpha 5 \beta 1$ or $\alpha v \beta 3$ induce apoptosis without causing cell detachment. This response, associated with the activation of protein kinase A (PKA), can be mimicked by increases in cAMP and by heterologous expression of constitutively active PKA and can be prevented by PKA inhibition. Apoptosis appears to be mediated by activation of the inducer caspase, caspase 8 , both in vitro in endothelial cells and in vivo in the chick chorioallantoic membrane. Together, these results strongly suggest that a pathway that links at least two different integrins to inhibition of PKA and protection from apoptosis plays an important role in blood vessel growth or maintenance. They also identify PKA as a potential new target for antiangiogenic therapies.

\section{Effects of integrin blockade and integrin deletion}

The first evidence that a specific integrin might play a critical role in pathologic angiogenesis came from studies of antibodies and small molecules that serve as antagonists of the integrin $\alpha v \beta 3(2,3)$. Those studies demonstrated that this integrin is induced in endothelial cells of angiogenic vessels and that reagents designed to block this integrin can be remarkably effective in preventing angiogenesis in several different models. Subsequent evidence suggested that reagents targeting another closely related integrin, $\alpha v \beta 5$, were similarly effective in a subset of angiogenic responses specifically dependent on the growth factor VEGF A (4). Furthermore, the integrin $\alpha 5 \beta 1-$ the central focus of the present paper by Kim et al. - has been shown to be induced in angiogenic vessels. As with the other integrins, targeting $\alpha 5 \beta 1$ effectively inhibits angiogenesis (5).

The simplest interpretation of these results, now no longer considered tenable, held that vascular development depends on active involvement of each of these integrins. Indeed, the phenotype of $\alpha 5$ subunit knockout mice seemed consistent with a critical requirement for $\alpha 5 \beta 1$ in this process (7) since these animals die at embryonic days 10-11 with severe defects in both embryonic and extra-embryonic vascular development. On the other hand, patients with the human disease Glanzmann thrombasthenia, many of whom carry null mutations in the integrin $\beta 3$ subunit, appear to be free of abnormalities in vascular development or angiogenesis. Still more compelling evidence undermining the simple model for integrin involvement emerged from careful study of mice expressing null mutations of a variety of $\alpha \mathrm{v}$ integrins. Mice lacking the $\alpha \mathrm{v}$ subunit (and therefore lacking $\alpha v \beta 3$, $\alpha v \beta 5$, and three other $\alpha \mathrm{v}$ heterodimers) show defects in vascular integrity, manifested by intracerebral and gastrointestinal hemorrhage (8). However, this phenotype appears to be largely explained by loss of the integrin $\alpha v \beta 8$ since many of the vascular defects in these animals also occur in $\beta 8$ subunit knockout mice (9), whereas mice lacking the $\beta 3(10)$, or $\beta 5$ subunits (11), or even both together (12) 
show no detectable defects in normal or pathologic vascular development. In fact, $\beta 3$ knockout and $\beta 3 / \beta 5$ double knockout mice demonstrate enhanced tumor angiogenesis, an effect that may be due to compensatory upregulation of the vascular endothelial growth factor receptor II in these animals (12). These observations have led some to suggest that the principal roles of $\alpha v \beta 3$ and $\alpha v \beta 5$ are not to enhance angiogenesis, as was previously proposed, but actually to inhibit it. If so, the profound inhibition of angiogenesis seen in vivo following integrin blockade with antibodies or small molecule antagonists clearly cannot be explained by simple loss of endothelial cell adhesion or integrin function.

Initial efforts to explain how integrin antagonists might induce endothelial apoptosis and inhibit angiogenesis focused on the phenomenon of anoikis, a process by which epithelial (13) or endothelial cells (14) rapidly undergo apoptosis following complete detachment from adhesive substrates (i.e., after loss of all signals from integrins). However, angiogenic vascular endothelial cells simultaneously express $\alpha v \beta 3$, $\alpha v \beta 5, \alpha 5 \beta 1$, as well as several other integrins, so it has been difficult to explain the potent effects of inhibiting any single integrin on angiogenesis by invoking loss of global input from integrins. However, the present study by Kim et al. (6) and a previously published study by Stupack et al. (15) suggest an alternative model. In both papers, individual integrins are shown to activate a caspase 8 -dependent pathway when they are expressed but not ligated. In this fashion, inhibitors of individual integrins can induce endothelial cell apoptosis even if the cells remain attached through ligation of other integrins.

It thus appears that nature has evolved an elegant system of checks and balances that allows for precise spatial regulation of vascular growth through coordinate regulation of several integrins and their ligands, ensuring that endothelial cells die if they migrate or spread into inappropriate environments. At the same time, this system is robust enough to preserve the critical function of vascular development even in the face of inactivating mutations of one or more of the integrins involved in this process. Because induction of apoptosis appears to depend on the presence of unligated integrin, endothelial cells lacking any individual integrins are not subject to apoptosis.

Genetic evidence suggests that this model too may be too simple to account for the significance of integrins in vascular growth and survival. In particular, endothelial $\alpha 5 \beta 1$ may not be dispensable since mice lacking this integrin have marked defects in vascular development. It is conceivable that $\alpha 5 \beta 1$ plays distinct roles in embryonic vasculogenesis and pathologic angiogenesis, but information about such differences must await studies in mice expressing conditional null mutations of the $\alpha 5$ subunit.

\section{Therapeutic targeting \\ of vascular integrins}

Although recent studies have forced researchers to revise their understanding of how the blockade of integrins affects angiogenesis, $\alpha 5 \beta 1, \alpha v \beta 3$, and $\alpha v \beta 5$ continue to offer viable drug targets. Attempting to target these integrins or downstream effectors, like PKA, to promote apoptosis in the treated cell remains an attractive strategy to enhance the killing of endothelial cells in angiogenic blood vessels. The recent report that $\alpha v \beta 3$-mediated targeting of an apoptosis-inducing mutant form of Raf kinase to tumor endothelium causes substantial regression of tumors in mice (16) should further spur efforts in this area. Just as endothelial cells bene- fit from the variety of integrins they can engage to regulate survival or death in response to their local environment, investigators aiming to prevent the pathological consequences of angiogenesis appear to have multiple molecular targets available.

1. Hynes, R.O. 1992. Integrins: versatility, modulation, and signaling in cell adhesion. Cell. 69:11-25.

2. Brooks, P.C., et al. 1994. Integrin $\alpha v \beta 3$ antagonists promote tumor regression by inducing apoptosis of angiogenic blood vessels. Cell. 79:1157-1164.

3. Brooks, P.C., Clark, R.A., and Cheresh, D.A. 1994 Requirement of vascular integrin $\alpha v \beta 3$ for angiogenesis. Science. 264:569-571.

4. Freidlander, M., et al. 1995. Definition of two angiogenic pathways by distinct $\alpha v$ integrins. Science. 270:1500-1502.

5. Kim, S., Bell, K., Mousa, S., and Varner, J.A. 2000. Regulation of angiogenesis in vivo by ligation of integrin $\alpha 5 \beta 1$ with the central cell binding domain of fibronectin. Am. J. Pathol. 156:1345-1362.

6. Kim, S., Bakre M., Yin, H., and Varner, J.A. 2002 Inhibition of endothelial cell survival and angiogenesis by protein kinase A. J. Clin. Invest. 110:933-941. doi:10.1172/JCI200214268.

7. Yang, J.T., Rayburn, H., and Hynes, R.O. 1993. Embryonic mesodermal defects in $\alpha 5$ integrindeficient mice. Development. 119:1093-1105.

8. Bader, B.L., Rayburn, H., Crowley, D., and Hynes, R.O. 1998. Extensive vasculogenesis, angiogenesis, and organogenesis precede lethality in mice lacking all $\alpha_{\mathrm{v}}$ integrins. Cell. 95:507-519.

9. Zhu, J., et al. 2002. beta8 integrins are required for vascular morphogenesis in mouse embryos. Development. 129:2891-2903.

10. Hodivala-Dilke, K.M., et al. 1999. $\beta 3$-integrindeficient mice are a model for Glanzmann thrombasthenia showing placental defects and reduced survival. J. Clin. Invest. 103:229-238.

11. Huang, X.Z., Griffiths, M., Wu, J.F., Farese, R.F., Jr., and Sheppard, D. 2000. Impaired cell migration and adhesion but normal development in $\beta 5$ deficient mice. Mol. Cell. Biol. 20:755-759.

12. Reynolds, L.E., et al. 2002. Enhanced pathologi$\mathrm{cal}$ angiogenesis in mice lacking $\beta 3$ integrin or $\beta 3$ and $\beta 5$ integrins. Nat. Med. 8:27-34.

13. Frisch, S.M., and Francis, H. 1994. Disruption of epithelial cell-matrix interactions induces apoptosis. J. Cell Biol. 124:619-626.

14. Meredith, J.E., Jr., Fazeli, B., and Schwartz, M.A 1993. The ECM as a cell survival factor. Mol. Biol. Cell. 4:953-961.

15. Stupack, D.G., Puente, X.S., Boutsaboualoy, S., Storgard, C.M., and Cheresh, D.A. 2001. Apoptosis of adherent cells by recruitment of caspase- 8 to unligated integrins. J. Cell Biol. 155:459-470.

16. Hood, J.D., et al. 2002. Tumor regression by targeted gene delivery to the neovasculature. Science. 296:2404-2407. 